\title{
The area under the waveform of electromyography for monitoring the external branches of the superior laryngeal nerve during thyroid surgery
}

\author{
Yishen Zhao ${ }^{1}$, Zihan Zhao ${ }^{1}$, Tie Wang ${ }^{1}$, Daqi Zhang ${ }^{1}$, Gianlorenzo Dionigi ${ }^{2}$, Hui Sun ${ }^{1}$ \\ ${ }^{1}$ Division of Thyroid Surgery, China-Japan Union Hospital of Jilin University, Jilin Provincial Key Laboratory of Surgical Translational Medicine, \\ Jilin Provincial Precision Medicine Laboratory of Molecular Biology and Translational Medicine on Differentiated Thyroid Carcinoma, Changchun, \\ China; 'Division for Endocrine and Minimally Invasive Surgery, Department of Human Pathology in Adulthood and Childhood 'Gaetano Barresi', \\ University Hospital G. Martino, University of Messina, Messina, Italy \\ Contributions: (I) Conception and design: All authors; (II) Administrative support: H Sun, G Dionigi; (III) Provision of study materials or patients: \\ H Sun, Y Zhao; (IV) Collection and assembly of data: Y Zhao, Z Zhao; (V) Data analysis and interpretation: Y Zhao; (VI) Manuscript writing: All \\ authors; (VII) Final approval of manuscript: All authors. \\ Correspondence to: Hui Sun. 126 Xiantai Street, Changchun 130033, China. Email: s_h@jlu.edu.cn.
}

Background Injuries to the external branches of the superior laryngeal nerve (EBSLN) are difficult to identify during thyroidectomy. Monitoring the low amplitudes of the EBSLN during surgery has proven to be complicated. Therefore a new parameter, the area under the waveform (AUW), was designed to improve monitoring efficiency. The aim of this study was to determine the effectiveness of using AUW to monitor the EBSLN during thyroidectomy.

Methods: A total of 927 patients and 927 recurrent laryngeal nerves (RLN) and EBSLNs who underwent monitored unilateral thyroidectomy were included. Standardized intraoperative neuromonitoring procedures were followed. RLN injuries were confirmed using stroboscopic laryngoscopy after surgery. EBSLN injuries were identified by means of observing changes in cricothyroid muscle (CTM) twitches when stimulated. Amplitude and AUW changes were assessed using statistical analysis. The correlations between AUW and amplitude were verified using the ratio of the two parameters to determine any decreases that were observed in the number of nerve injury cases. The receiver operating characteristic (ROC) curve was used to estimate the prediction of nerve injury.

Results: The overall injury rates of the RLN and EBSLN were $3.56 \%$ and $2.05 \%$, respectively. The AUW for the RLN and EBSLN presented linear correlations with amplitude. In the RLN injury cases, no significant difference was observed between the two parameters $(\mathrm{P}>0.05)$. In the cases with EBSLN injuries, the AUW decreased $64.5 \% \pm 14.1 \%$, on average, which was a greater decrease compared to that observed for amplitude $49.7 \% \pm 22.2 \%(\mathrm{P}<0.0001)$. The summary of the EBSLN injury prediction included, amplitude $v s$. AUW, AUC: 0.918 vs. 0.994; $\mathrm{P}<0.0001$, and Youden's index: $31.54 \%$ vs. $49.58 \%$.

Conclusions: The AUW demonstrated consistency with the change in amplitude, and the observed changes were significant. The use of the AUW allowed successful predictions for both RLN and EBSLN nerve injuries. Also, the sensitivity of AUW was greater than amplitude for predicting EBSLN injuries.

Keywords: Thyroid surgery; intraoperative neuromonitoring; external branch of superior laryngeal nerve; morbidity; nerve injury

Submitted Jun 19, 2020. Accepted for publication Sep 30, 2020.

doi: $10.21037 /$ gs-20-570

View this article at: http://dx.doi.org/10.21037/gs-20-570 


\section{Introduction}

Intraoperative neural monitoring (IONM) is a supplementary technology used to identify the external branch of the superior laryngeal nerve (EBSLN) and assess nerve function during thyroid surgery (1-3). Monitoring of the EBSLN is gradually being recognized as having an essential role in thyroid surgery (4). In 2013, the International Neuromonitoring Study Group (INMSG) released the first guidelines to standardize the techniques used to monitor the EBSLN (5). Nevertheless, despite the importance of the INMSG guidelines, the techniques currently used for monitoring the EBSLN during surgery are still in the early phases of development. EBSLN monitoring is especially challenging due to specific intrinsic electromyographic (EMG) nerve characteristics. The amplitude of the EBSLN is lower and presents more significant individual variations than the recurrent laryngeal nerve (RLN). Dionigi et al. assessed EMG profiles in 400 EBSLNs using the Cernea classification and reported that the mean amplitudes for types $1,2 \mathrm{~A}$, and $2 \mathrm{~B}$ were $259 \pm 67 \mu \mathrm{V}$ [180-421], $321 \pm 79 \mu \mathrm{V}$ [192-391], and $371 \pm 38$ $\mu \mathrm{V}$ [200-551], respectively (6,7). Barczynski et al. found that the mean EBSLN amplitude was $249 \pm 144 \mu \mathrm{V}$ (8). Potenza $e t$ al. verified that the mean amplitude value was $269 \pm 176 \mu \mathrm{V}$ in $73 \mathrm{EBSLNs}$ (9). Our study group previously identified that the EBSLN plateau stimulating current was higher than that of the RLN (10). Furthermore, 70-100\% of the EBSLNs were observed to induce EMG signals via an endotracheal (ET) monitoring tube, which may be related to the sensitivity of the EMG signal acquisition of the special monitoring equipment that was used $(5,8,9)$.

Since the EBSLN branches enter the cricothyroid muscle (CTM) and then extend into the larynx to innervate the thyroarytenoid muscle, EBSLN depolarization potentials can be transmitted directly to the vocal cord to generate an artifactual EMG signal (11). Moreover, the EMG latency is extremely short because of the short transmission distance $(8,9)$. Simultaneously, the EBSLN can cause a vocal cord EMG response through the anastomotic branch of the RLN, and induce an EMG latency that is slightly longer (5).

To refine the technology and technique of EBSLN monitoring, the area under the waveform (AUW, as to distinguish from the statistical acronym AUC, or area under the curve) was used during monitored thyroidectomy and was examined on more than 900 EBSLN nerves that were at risk (NAR) during surgery (Figure 1).

We present the following article following the Materials
Design Analysis Reporting (MDAR) checklist (available at http://dx.doi.org/10.21037/gs-20-570).

\section{Methods}

\section{Study design, setting, and source population}

Included patients were recruited from a larger cohort of evaluated, treated, and received follow-up care at the Division of Thyroid Surgery of the China-Japan Union Hospital at Jilin University. Data were collected between December 2018 and October 2019. Surgery was performed by endocrine surgeons with extensive experience in nerve monitoring. The study was conducted following the Declaration of Helsinki (as revised in 2013) and was approved by the Institutional Review Board of ChinaJapan Union Hospital of Jilin University (Changchun City, China; approval no. 20181203). Individual consent for this retrospective analysis was waived.

\section{Eligibility criteria}

\section{Inclusion criteria}

Patients who were scheduled for monitored open unilateral thyroidectomy for thyroid cancer and goiter were assessed preoperatively using ultrasound or computed tomography (CT) imaging and aspiration cytology. The inclusion criteria were as follows:

(I) Patients with high RLN injury risk, including those with thyroid cancer, large benign thyroid tumors $>100 \mathrm{~g}$, a thyroid gland $>240 \mathrm{~g}$, or those undergoing a repeat surgery (3).

(II) Patients with high EBSLN injury risk, including those with thyroid cancer, large benign tumors $>100 \mathrm{~g}$ in the superior pole of the thyroid gland, a thyroid gland $>240 \mathrm{~g}$, undergoing a repeat surgery, or patients whose upper edge of the superior pole of the thyroid was higher than the upper edge of the thyroid cartilage (5).

Thyroid quality and volume were estimated preoperatively using ultrasonography. Thyroid ultrasounds were performed using a 4.8-11 MHz linear probe.

\section{Exclusion criteria}

Patients were excluded from the study if they exhibited any of the following conditions: vocal cord paralysis before surgery, the surgical plan was intraoperatively changed to total thyroidectomy, both the RLN and EBSLN 
A
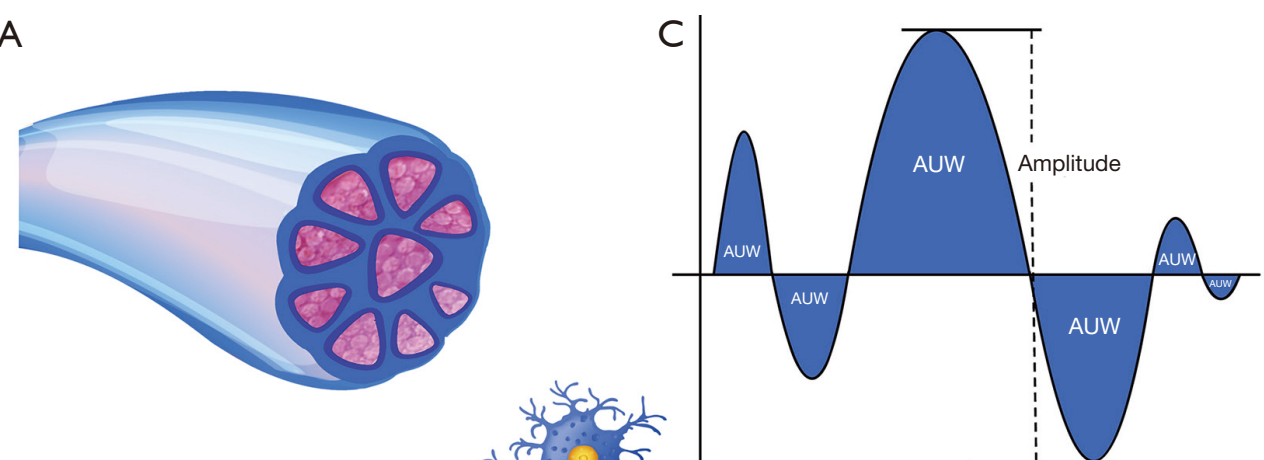

B
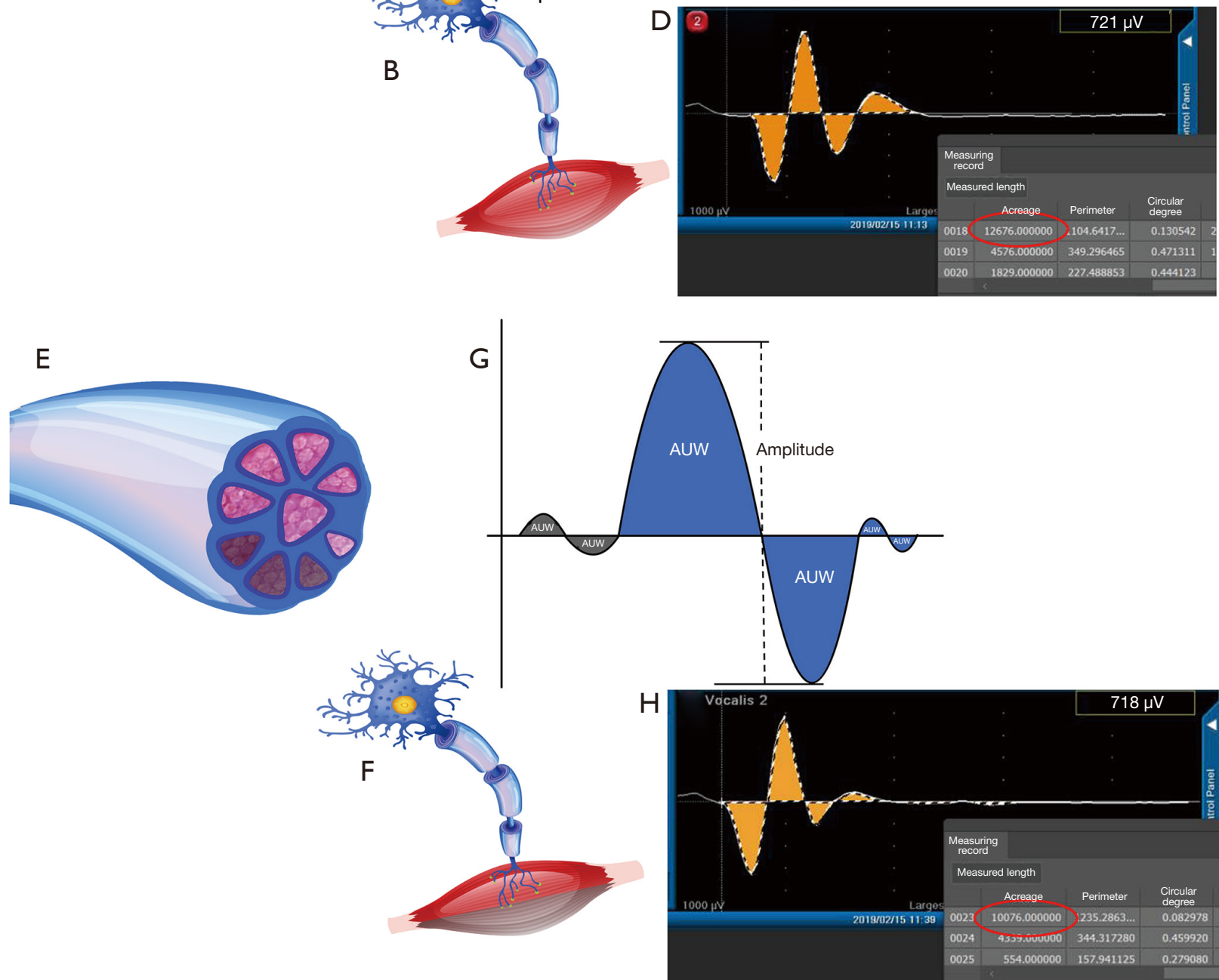

Figure $1 \mathrm{~A}$ schematic diagram of the new parameter, AUW. Upper figures (A,B,C,D) show the depolarization of the affected muscle that is innervated by a normal nerve after electrical stimulation and the electromyogram that is formed. (A) A section of a compound motor nerve. (B) A motor endplate and the innervated muscle. (C) A diagram of the amplitude pattern and the AUW. (D) The interface of the intraoperative neuromonitoring, including the AUW (12,676 px) that was obtained through processing by the graphic software. Lower figures (E,F,G,H) show the depolarization of the affected muscle that is innervated by a partially injured nerve after electrical stimulation and the electromyogram that is formed. (E) A section of a compound motor nerve, where the dark part represents the injured parts of the nerve fiber. (F) The motor endplate and affected muscle. The dark part represents the injured muscle fiber. (G) A diagram of the pattern of the amplitude and the AUW. The grey part represents the altered AUW, and the amplitude did not change. $(\mathrm{H})$ The interface of the intraoperative neuromonitoring, including the AUW, which was decreased (10,076 px, 20\% decreased) and the amplitude that did not change. AUW, area under waveform. 
were injured simultaneously during surgery, endoscopic surgery was used, patients $<18$ years of age, and those with incomplete/unavailable data.

The eligibility mentioned above criteria applies only to this study.

\section{IONM setup and standards}

IONM was provided in an intermittent application mode. Nerve monitoring was performed according to the standards established for equipment set up, induction and maintenance of anesthesia, verification testing for proper tube positioning, and EMG definitions (3). ET tube-based surface electrodes were applied (Trivantage EMG tube, Medtronic, Jacksonville, Florida, USA) and connected to audio and visual IONM systems (NIM-Response 3.0, Medtronic, Jacksonville, Florida, USA). The EBSLNs were stimulated using single-use, incrementing Prass stimulating probes, monopolar, with a standard flexible tip (product n. 8225490, Medtronic, Jacksonville, Florida, USA) at $100 \mathrm{~ms}$ impulse duration and a $4 \mathrm{~Hz}$ frequency.

\section{Surgical procedures}

Surgery was standardized according to RLN and EBSLN INMSG guidelines $(3,5)$. For visually identified EBSLNs, a $1.0 \mathrm{~mA}$ current was preferred. For EBSLN mapping and dissection, a higher current value was used (2-3 mA). In this study, the EBSLN was identified and monitored using the following procedure:

(I) Dissection. Defined anatomical structures, and in particular, the sternothyroid-laryngeal-superior pole triangle was used. Gentle traction was applied to the superior thyroid lobe in the lateral and caudal direction to obtain adequate exposure of the sternothyroid-laryngeal triangle and the pars recta and pars obliqua of the CTM. Meticulous dissection of the non-perfused area between the CTM and the upper pole of the thyroid was performed. A precise surgical technique is necessary to preserve the CTM and the EBSLN, with clear operational control and exposure of the sternothyroid-laryngeal triangle. Transverse sectioning of the cranial portion of the sternothyroid muscle was performed with caution due to the possible proximity of the EBSLN.

(II) Mapping. The lateral position of the superior pole of the thyroid was maintained throughout all EBSLN identifications, confirmations, and monitoring. The
EBSLN was mapped using $1.5-2.0 \mathrm{~mA}$ current in the sternothyroid-laryngeal triangle, while simultaneously observing for the nerve, a positive CTM twitch, and an EMG response.

(III) Nerve confirmation. Visual (nerve and CTM twitch) and electrical (clear biphasic waveform with recognizable amplitude) characteristics of the EBSLN were assessed before individual ligation of the STV branches. It was essential to test the most cranial portion of the nerve and to stimulate the segment above the dissected region of the nerve during superior pole management so that the nerve could be adequately tested during this procedure. The EBSLN EMG signal (S1) was obtained using a $1.0 \mathrm{~mA}$ current. Some EBSLNs were located deep to the fascia of the inferior constrictor muscle. These particular nerves were located and mapped using $2.0 \mathrm{~mA}$ instead of using intramuscular dissection.

(IV) Functional evaluation and classification. After complete STV ligation, thyroidectomy, and hemostasis, the cranial aspect of the exposed EBSLN was stimulated with $1.0 \mathrm{~mA}$ (S2). The movement of the CTM to the electric stimulation of $\mathrm{S} 2$ relative to that of $\mathrm{S} 1$ was used to evaluate whether EBSLN injury had occurred, including significant weakening or total disappearance.

\section{Follow-up}

Stroboscopic laryngoscopy (light source, ATMOS, GER; fiber laryngoscope, PENTAX, JPN) was offered one day before and one day after surgery. The same surgeon performed all of the laryngoscope exams. Any abnormal vocal cord movement was observed and documented (12).

\section{Outcomes}

Necessary information, including sex and age, was obtained before surgery. The EMG waveform images, including all of the data parameters recorded for each NAR, comprised of the EBSLN (S1, S2), RLN (R1, R2), VN (V1, V2), AUW, and amplitude determinations $(3,5,13)$. After the surgery, the AUW was recorded using graphic processing software (Adobe Photoshop CC 2015, Adobe, USA), with pixels (px) as the basic unit (Figure 1C,D,G,H). The pixel values corresponding to each amplitude data were recorded, and statistical analyses were conducted postoperatively. 
The EMG profiles were compared with the pre- and postoperative stroboscopic laryngoscopic evaluations (L1, L2) at 48 hours before surgery and on the first postoperative day $(3,5,13)$. Pre-, intra-, and postoperative needle EMGs of the CTM were not performed.

\section{Statistical analysis}

The sample size was estimated based on the principle of detecting a difference of $-50 \mu \mathrm{V}$ between the EBSLN subtypes. The power was set to $90(\beta=0.9)$, and the standard deviation of the means was set at -10 , with a $90 \%$ probability at $\mathrm{P}<0.05$ [using power curve and sample size tools for oneway analysis of variance (ANOVA)]. Therefore, a sample size of approximately 900 NAR per group should have provided $90 \%$ power to detect an amplitude difference of $50 \mu \mathrm{V}$ between the different NAR subtypes.

Statistical methods were used to verify linear correlations between the amplitude and the AUW in the comprehensive monitoring data of the RLNs and EBSLNs. The validity of the AUW in providing a warning of RLN and EBSLN injuries and the consistency between the AUW and amplitude was verified by observing a decrease in the ratio of these two parameters among the cases with nerve injuries. We also determined the monitoring characteristics of the AUW in RLN and EBSLN injuries and the ability of the AUW to provide early warning of nerve injury.

SPSS version 23 software (IBM, Armonk, NY, USA) was used for all statistical analyses. Measurement data with normal distributions were expressed as mean \pm standard deviation. Differences between the groups were compared using the Student's $t$-test. The Pearson correlation coefficient (r) was assessed to determine the correlation between the two parameters. The receiver operating characteristic (ROC) curve method was used to evaluate the predictive ability of the AUW and the amplitude for RLN and EBSLN injury. The AUC was used to evaluate the ability of the two parameters to provide warning of nerve injury. Youden's index (14) was used to calculate the cutoff value for the percentage of the decline of the two parameters for the warning of nerve injury. All tests were two-tailed, with the level of significance set at $\mathrm{P}<0.05$.

\section{Results}

\section{Clinical-pathological characteristics}

A total of 1,006 patients were involved in the recruitment, and 927 cases were included in the final analysis. Seventynine cases were excluded, resulting in an exclusion rate of $7.9 \%$. Of the included patients, 235 were males, and 692 were females, with a mean age of $41.6 \pm 24.4$ years. Among these patients, 817 cases with thyroid cancer were treated with unilateral thyroidectomy, isthmectomy, and central lymph node dissection. The remaining 110 cases with benign goiter were treated with unilateral thyroidectomy only.

\section{Nerves at risk (NAR)}

All 927 EBSLNs and RLNs were identified and monitored. Through electrical stimulation of the EBSLN at the superior thyroid pole, the CTM movement was triggered. Thirty-three cases $(3.5 \%)$ of RLN injury were confirmed using stroboscopic laryngoscopy, and 19 cases $(2.1 \%)$ of EBSLN injury were identified using electrical stimulation of the EBSLN during surgery.

\section{EMG data and stroboscopic observations}

We compared the R2 and R1 signals and observed that the RLN AUW overall data were consistent with the difference in amplitude. Pearson correlation of the RLN data indicated a linear correlation $(\mathrm{r}=0.612, \mathrm{P}<0.0001)$. We also compared the S2 and S1 signals and found that the EBSLN AUW overall data were consistent with the difference in amplitude. Pearson correlation of the EBSLN overall data indicated a linear correlation $(\mathrm{r}=0.719, \mathrm{P}<0.0001)$ (Figure 2A,B).

For the RLN and EBSLN injury data, the AUW showed a linear relationship with the amplitude change $\left(\mathrm{r}_{\mathrm{RLN}}=0.563\right.$, $\mathrm{P}=0.001$ and $\mathrm{r}_{\mathrm{EBSLN}}=0.800, \mathrm{P}<0.0001$ ) (Figure $\left.3 A, B\right)$.

We also assessed the decrease in nerve injury data and found that the initiation of the AUW change was the same as the amplitude. However, the decline in the AUW was significantly more significant compared to the amplitude. Furthermore, the absolute value of the change in AUWs was markedly more significant than that of the amplitude change (RLN and EBSLN injury data, $\mathrm{P}<0.0001)$.

In cases with RLN injury, laryngoscopy revealed the abnormal movement of the vocal cords (weak or absent). On average, the amplitude decreased by $84.8 \% \pm 14.7 \%$ (with a range of $47.4-100 \%$ ), and the AUW decreased by $83.0 \% \pm 17.9 \%$ (with a range of $28.7-100 \%$ ). No significant difference was observed between these two parameters $(\mathrm{P}>0.05)$. Thus, both parameters were useful in monitoring 

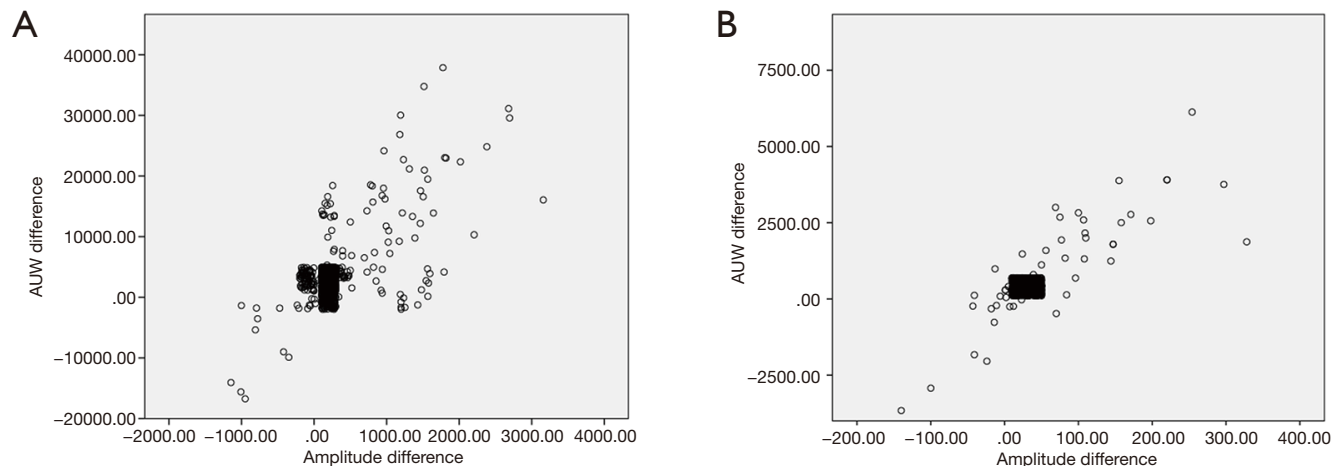

Figure 2 The Pearson correlation of the AUW and the amplitude difference of RLN and EBSLN, including all the data. (A) The RLN data that show a linear correlation, $\mathrm{r}=0.612$, and $\mathrm{P}<0.0001$. (B) The EBSLN data showing a linear correlation, $\mathrm{r}=0.719$ and $\mathrm{P}<0.0001 . \mathrm{AUW}$, area under waveform; RLN, recurrent laryngeal nerve; EBSLN, external branch of the superior laryngeal nerve.
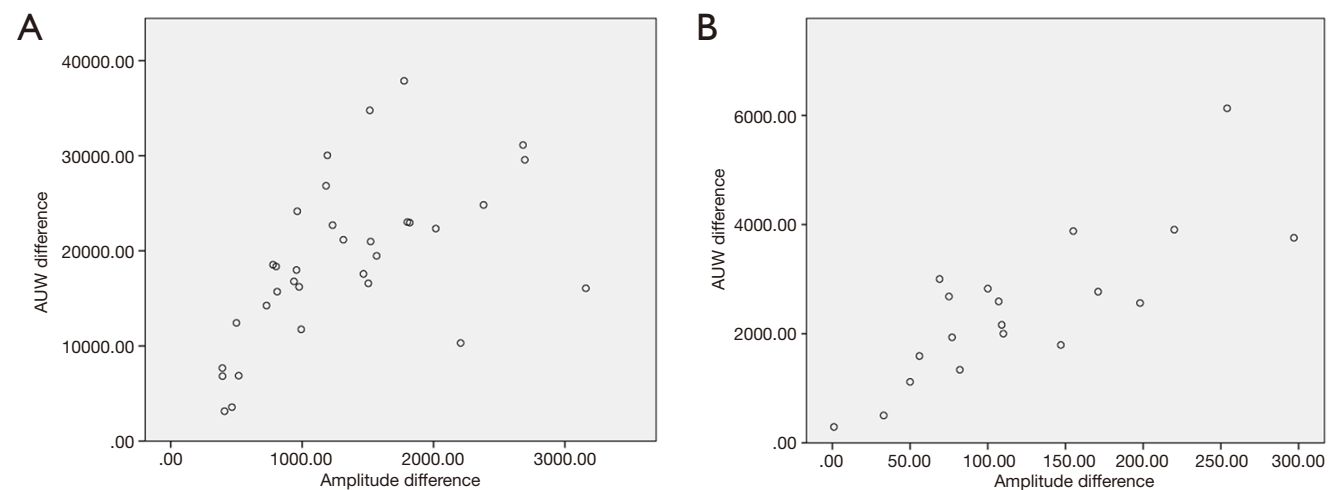

Figure 3 The Pearson correlation of the AUW and the amplitude difference for the RLN and EBSLN, when injury data were included. (A) $\mathrm{r}_{\mathrm{RLN}}=0.563, \mathrm{P}=0.001$. (B) $\mathrm{r}_{\mathrm{EBSLN}}=0.800, \mathrm{P}<0.001$. AUW, area under waveform; RLN, recurrent laryngeal nerve; EBSLN, external branch of the superior laryngeal nerve.

\section{RLN injury (Figure 4A).}

In cases with EBSLN injury, no significant changes in vocal cord movement were observed with laryngoscopy. However, stroboscopic laryngoscopy revealed that the mucosal waves were either weak or unchanged. On average, the amplitude decreased by $49.7 \% \pm 22.2 \%$ (with a range of $1.4-100 \%$ ), and the AUW decreased by $64.5 \% \pm 14.1 \%$ (with a range of $51.6-100 \%)$. The decrease in the AUW was significantly more significant than the decrease in amplitude $(\mathrm{P}<0.0001)$ (Figure 4B).

The decreased percentage of ROC curves of AUW and amplitude in RLN and EBSLN injury

ROC curves were used to warn and predict injury to the RLNs. RLN injury was warned by the decreased percentage in the amplitude vs. AUW (the AUC was 0.998 vs. 0.995;
$\mathrm{P}=0.001$ vs. $\mathrm{P}=0.003$; Youden's index was $46.47 \%$ vs. $50.50 \%$ ) (Figure 5A). Similarly, EBSLN injury was also warned by the decreased percentage in the amplitude $v s$. AUW (AUC was 0.918 vs. $0.994 ; \mathrm{P}<0.0001$ vs. $\mathrm{P}<0.0001$; Youden's index was $31.54 \%$ vs. $49.58 \%$ ) (Figure $5 B$ ).

\section{Discussion}

In this study, the concept of the AUW was used to evaluate the function of RLNs and EBSLNs for the first time. The AUW also was used to provide warning of intraoperative nerve injury and predict postoperative vocal cord movements. Using statistical analyses, we found that the AUW was correlated with the EMG amplitude (which is the conventionally used parameter), demonstrating that the AUW was useful for assessment. We further analyzed 

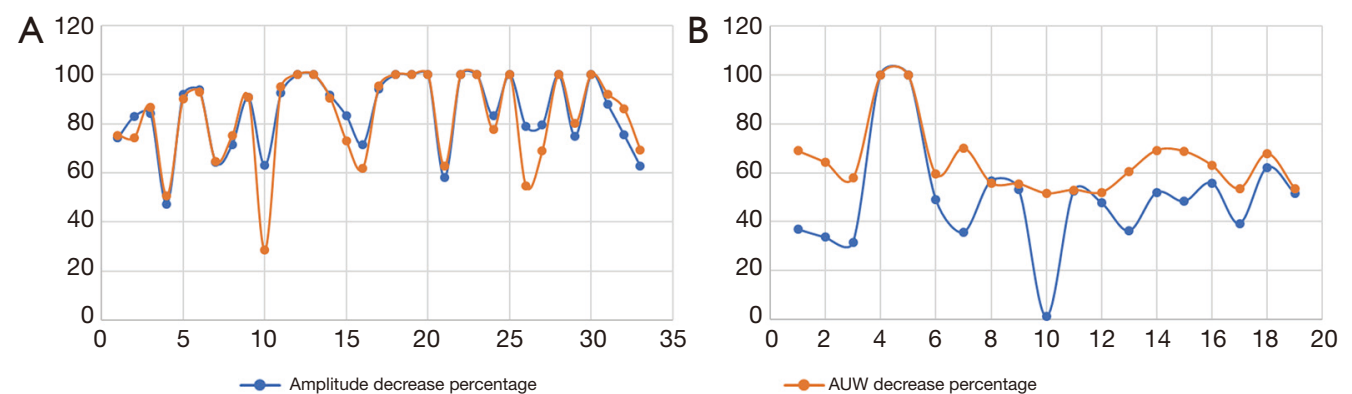

Figure 4 The difference between the AUW and the percentage of the amplitude decrease for the RLN and the EBSLN when considering only the injury data. (A) The RLN injury cases; (B) the EBSLN injury cases. AUW, area under waveform; RLN, recurrent laryngeal nerve; EBSLN, external branch of the superior laryngeal nerve.


Figure 5 ROC curves for injury prediction for the RLNs and EBSLNs using the AUW and the amplitude, and including all the data. (A) The ROC curve for RLN injury prediction using the AUW and amplitude. (B) The ROC curve for EBSLN injury prediction using the AUW and amplitude. ROC, receiver operating characteristic curve; AUW, area under waveform; RLN, recurrent laryngeal nerve; EBSLN, external branch of the superior laryngeal nerve.

the characteristics and monitoring advantages of the AUW. The ROC curve, combined with the results of stroboscopic laryngoscopy and the movement of the CTM induced by EBSLN electrical stimulation, were used to evaluate the ability of the two parameters to provide warning of RLN and EBSLN injury.

Figure 1 shows the basic principles of the AUW. Similar to the formation of a myocardial EMG, the RLN or EBSLN waveform image displayed by the EMG in IONM was the composite waveform generated by the depolarization of multiple groups laryngeal muscle fibers stimulated by the receiving electrode $(15,16)$. The electrical current stimulates all of the muscle fibers innervated by a compound motor nerve, and theoretically, small differences would exist in the time course of depolarization. Therefore, we assumed that a specific injury might cause one or several muscle fibers to lose their innervation, either temporarily or permanently. However, not all nerve injuries would lead to the dysfunction of the innervated muscle fibers at precisely the same time, especially in cases of minor injuries (such as nerve traction injury). The amplitude depends on the potential difference produced by the simultaneous depolarization of the stronger muscle fibers. However, this does not reflect the function of all the muscle fibers that are controlled by a compound nerve.

The AUW differs from amplitude in principle. The AUW represents the ability to depolarize all the muscle fibers controlled by a compound motor nerve at different times. When a portion of the compound nerve fibers that innervate muscle fibers with low potential are damaged, 
the weak or absent potential of these muscle fibers does not affect the change in amplitude, but it will change the AUW. Therefore, the AUW can accurately reflect the depolarization of all the motor muscle fibers stimulated by the receiving electrode in the larynx. Although the amplitude does not change, the movement of the whole muscle decreases. This is more apparent with a compound nerve, such as the EBSLN, which has low potential and wide distribution (Figure 1).

Evaluation of vocal cord movement using laryngoscopy is considered the benchmark in assessing intraoperative RLN injury and has been verified in many previous studies (17-20). In this study, the results of laryngoscopy were used as a diagnostic criterion to evaluate RLN function before and after surgery. However, there is currently no effective method to evaluate EBSLN injury immediately after surgery. In this study, all EBSLNs were detected using 0.5-2 mA electrical stimulation (S1) during the surgical manipulation of the superior pole of the thyroid gland. The area of EBSLN innervation could be determined by the movement of the CTM upon stimulation of the EBSLN. In some cases, the area of innervation was invaded by a tumor or was damaged by activation of the energy-based instruments. In these situations, the EBSLN injury was confirmed by re-stimulating the area of the superior pole (S2) and noting that the movement of the CTM was weak or absent.

The RLN injury rate observed in this study was similar to other reports in the literature $(17,21,22)$. However, the injury rate for the EBSLN was lower than previous reports $(0-58 \%$, with $3.8 \%$ permanent injury) $(4,21,23,24)$. This observation may be related to the lack of long-term postoperative follow-up assessment. The intraoperative EBSLN injury could not be diagnosed accurately with postoperative stroboscopic laryngoscopy. In cases with EBSLN injury in this study, only some of the patients reported a sensation of the presence of a foreign body when swallowing, fatigue after vocalizing for a long time, or occasional choking. The abnormal vocal symptoms were not significant.

In our overall data comparison, the AUW was consistent with the amplitude change, and the two parameters displayed a significant linear correlation. In cases of injury to both nerves, we observed a notable linear correlation between the AUW and amplitude. These results indicate that the AUW exhibits a similar trend to the classical amplitude parameters concerning intraoperative monitoring ability. The AUW also has the capacity for real-time intraoperative evaluation of nerve function. However, we observed that the change in the absolute value of the AUW was considerably more significant than the amplitude. Concerning nerve injury, when the amplitude decreased, the absolute value of the AUW decreased by a more significant margin. This was especially true in cases of EBSLN injury due to the low initial amplitude, which made it difficult to monitor the amplitude in real-time during surgery. However, the AUW allows for a more consistent quantitative evaluation of nerve function during surgery, allowing surgeons to have a better intraoperative assessment of nerve function.

We also observed cases with injuries to the RLN and EBSLN and found that the starting point for changes in amplitude and the AUW was the same. This is reasonable considering that the decrease in amplitude represents a weakening of the muscle fibers with the most significant change of potential difference in the EMG, and thus significant changes also would take place in the corresponding waveform. However, an essential alternative hypothesis to consider would be the effect of weakened muscle fibers that are only able to cause a small change in the potential difference in the EMG. In this case, the AUW will still show observable changes, while the amplitude would remain unchanged because the stronger muscle fibers would not have changed (Figure 1). Thus, this result would make the AUW a more comprehensive and accurate indicator of the function of all the innervated muscle fibers in the larynx.

It has been reported that as the decrease in the range of parameters becomes larger, the observed changes in nerve function become more accurate $(22,25,26)$. In cases with RLN injury in this study, the decrease in the ratio of the two parameters was similar. The average amplitude decreased by $84.8 \% \pm 14.7 \%(47.4-100 \%)$, and the average AUC decreased by $83.0 \% \pm 17.9 \%$ (28.7-100\%). However, there was no statistical difference between the parameters ( $\mathrm{P}>0.05)$. Therefore, for the warning of RLN injury during surgery and vocal cord movement after surgery, both parameters are effective and can reflect nerve injury.

In cases with injury to the EBSLN, the decrease of the AUW was significantly more significant than the amplitude. In our analysis, we found that the amplitude of S1 and S2 in some cases of injury did not change significantly. This was observed even when the induced movement of the CTM was significantly weakened or absent, and the electric stimulation of S2 was relative to that of S1 (5). However, in these cases, the AUW still showed a more significant 
decline $(64.5 \% \pm 14.1 \%$ vs. $49.7 \% \pm 22.2 \%)$. The decrease in the range of the AUW was significantly more significant than the amplitude $(\mathrm{P}<0.05)$. Thus, we conclude that the sensitivity of the AUW is higher relative to amplitude for intraoperative evaluation of EBSLN injuries.

In this study, the AUC values for amplitude and the detection ability of two nerve injuries were sufficient to be diagnostic, especially for the RLN, where the amplitude vs. AUW was $99.8 \%$ vs. $99.5 \%$, respectively. This may relate to the absence of false-negative results in the data. In other words, these cases present insignificant changes in the intraoperative parameters, but nerve injury was present. The ROC curve analysis revealed that both the AUW and amplitude showed a high intraoperative diagnostic ability for both RLN and EBSLN injuries.

According to Youden's index, $46.47 \%$ was the cutoff value for the decrease in amplitude in the diagnosis of RLN injury. The cutoff value found in this study is close to the clinical warning value of $50 \%$ of the baseline amplitude but is somewhat more conservative than this value. The AUW also showed a similar level of diagnostic ability. The warning value for AUW for intraoperative RLN injuries was determined to be $50.50 \%$, which is closer to the median value of the range of warning indicators. Thus, the AUW indicator for RLN injury would be more convenient and intuitive for clinical use.

For the diagnosis of EBSLN injury, the Youden's index demonstrated that the cutoff value of the percentage of amplitude decrease was $31.54 \%$. This observation indicates that there were some previous misunderstandings in the monitoring of the EBSLN function. It is not advisable to use a decrease in amplitude of more than $50 \%$ as an early warning indicator for EBSLN injury. This is due to the low amplitude of the EBSLN $(60-300 \mu \mathrm{V})(5-9)$. Low initial amplitude and a small decrease representing the cutoff value make it difficult to evaluate EBSLN function during surgery effectively. Our results show that the amplitude of S2 relative to $\mathrm{S} 1$ changed more than $30 \%$, which may indicate impairment of EBSLN function. Therefore, monitoring the amplitude is a counter-intuitive and inconvenient method of evaluating EBSLN function during surgery. This observation is consistent with other practical problems that occur in clinical work.

Furthermore, the Younden's index for the AUW decrease was $49.58 \%$, close to the warning value for the conventional RLN monitoring. However, the value and range of change for the AUW were significant relative to the amplitude. Based on the statistical analysis of these data, we observed that, relative to amplitude, the AUW offered more advantages and practical effects for quantitative intraoperative monitoring of EBSLN function, extremely useful in clinical work.

In this study, stroboscopic laryngoscopy was also utilized. In addition to using the laryngoscope to observe vocal cord movement postoperatively, it had also been used in previous studies to diagnose RLN injury (17-20). However, stroboscopic laryngoscopy cannot be used to diagnose EBSLN injury. In cases of RLN injury, stroboscopic laryngoscopy has the same function as electronic laryngoscopy, which records the adduction or abduction of the vocal cord. The movement of mucosal waves on the surface of the vocal cord can also be observed. The mucosal waves on the surface of the vocal cord would disappear without mucosal rolling, even if a tremor does not disappear in cases where the vocal cord was fixed in the median or paramedian position. In cases with EBSLN injury, the symmetry of the vocal cord mucosal wave existed, but the bilateral fluctuation was weak compared with the preoperative values. Since the observed change was weak, it was challenging to diagnose intraoperative EBSLN injury, consistent with previous EBSLN research results (27).

Some limitations were present in this study. First, AUW measurements could not yet be displayed in real-time. In this study, we collected all of the real-time waveform and amplitude data in each surgery; however the real-time intraoperative AUW could not be restored. We aimed to design a parameter for more sensitive EBSLN integrity monitoring and examined its monitoring performance. As our results confirmed the efficiency of AUW for IONM, real-time AUW display can be programmed into both normal and continuous monitoring systems in the future, allowing for more accurate and instructive intraoperative decision-making.

Second, for cases with nerve injury, continuous intraoperative monitoring might have recorded the continuous changes in nerve function more effectively. If the continuous amplitude and the AUW with real-time changes in a specific nerve injury model could be verified using animal experiments, it would be more informative for earlier detection of nerve injuries.

Furthermore, direct EMG data (obtained via needle electrodes inserted into the CTMs or VMs) were not evaluated in this study and were not correlated with surface EMG data obtained from the ET tube. Investigating whether the AUW is a better warning indicator of nerve functioning in patients with an initially small amplitude at 
baseline presents an interesting avenue for future research. It is difficult to determine whether nerve injury will occur in every surgery; however, animal experiments can address these problems $(10,25,26,28)$. Therefore, it is necessary to design future animal experiments to verify the monitoring effect of the parameters of the AUW.

Also, this study only included a short-term evaluation of vocal cord movement and no long-term results. EBSLN injury was tested intraoperatively based on the subjective measure of lacking an S2 response in a positive S1 response. However, objective postoperative EMG via needle electrodes inserted into the CTM was not evaluated in this study. Therefore, it was not possible to determine whether the RLN or EBSLN injury was temporary or permanent.

Also, the recovery time and the degree of recovery from injury were not investigated. Future assessments using stroboscopic laryngoscopy may provide additional meaningful results $(29,30)$.

Lastly, the AUW in this study was calculated postoperatively, which is not suitable for intraoperative decision-making. Future work should focus on intraoperative software processing of this value for better surgical guidance.

\section{Acknowledgments}

Funding: None.

\section{Footnote}

Reporting Checklist: The authors have completed the MDAR reporting checklist. Available at http://dx.doi.org/10.21037/ gs-20-570

Data Sharing Statement: Available at http://dx.doi. org/10.21037/gs-20-570

Peer Review File: Available at http://dx.doi.org/10.21037/gs20-570

Conflicts of Interest: All authors have completed the ICMJE uniform disclosure form (available at http://dx.doi. org/10.21037/gs-20-570). The authors have no conflicts of interest to declare.

Ethical Statement: The authors are accountable for all aspects of the work in ensuring that questions related to the accuracy or integrity of any part of the work are appropriately investigated and resolved. The study was conducted in accordance with the Declaration of Helsinki (as revised in 2013). The study was approved by the Institutional Review Board of China-Japan Union Hospital of Jilin University (Changchun City, China; approval no. 20181203) and individual consent for this retrospective analysis was waived.

Open Access Statement: This is an Open Access article distributed in accordance with the Creative Commons Attribution-NonCommercial-NoDerivs 4.0 International License (CC BY-NC-ND 4.0), which permits the noncommercial replication and distribution of the article with the strict proviso that no changes or edits are made and the original work is properly cited (including links to both the formal publication through the relevant DOI and the license). See: https://creativecommons.org/licenses/by-nc-nd/4.0/.

\section{References}

1. Roy N, Smith ME, Dromey C, et al. Exploring the phonatory effects of external superior laryngeal nerve paralysis: an in vivo model. Laryngoscope 2009;119:816-26.

2. Roy N, Barton ME, Smith ME, et al. An in vivo model of external superior laryngeal nerve paralysis, laryngoscopic findings. Laryngoscope 2009;119:1017-32.

3. Randolph GW, Dralle H, Abdullah H, et al. Electrophysiologic recurrent laryngeal nerve monitoring during thyroid and parathyroid surgery: international standards guideline statement. Laryngoscope 2011;121 Suppl 1:S1-16.

4. Barczyński M, Randolph GW, Cernea C, et al. International survey on the identification and neural monitoring of the EBSLN during thyroidectomy. Laryngoscope 2016;126:285-91.

5. Barczyński M, Randolph GW, Cernea CR, et al. External branch of the superior laryngeal nerve monitoring during thyroid and parathyroid surgery, International Neural Monitoring Study Group standards guideline statement. Laryngoscope 2013;123:S1-14.

6. Dionigi G, Kim HY, Randolph GW, et al. Prospective validation study of Cernea classification for predicting EMG alterations of the external branch of the superior laryngeal nerve. Surg Today 2016;46:785-91.

7. Cernea CR, Ferraz AR, Nishio S, et al. Surgical anatomy of the external branch of the superior laryngeal nerve. Head Neck 1992;14:380-3.

8. Barczynski M, Konturek A, Stopa M, et al. Randomized 
controlled trial of visualization versus neuromonitoring of the external branch of the superior laryngeal nerve during thyroidectomy. World J Surg 2012;36:1340-7.

9. Potenza AS, Phelan EA, Cernea CR, et al. Normative intra-operative electrophysiologic waveform analysis of superior laryngeal nerve external branch and recurrent laryngeal nerve in patients undergoing thyroid surgery. World J Surg 2013;37:2336-42.

10. Zhao Y, Li C, Liu X, et al. Investigation on EMG Profiles of the Superior Laryngeal Nerve in a In Vivo Porcine Model. J Invest Surg 2020;33:596-604.

11. Uludag M, Aygun N, Kartal K, et al. Innervation of the human posterior cricoarytenoid muscle by the external branch of the superior laryngeal nerve. Head Neck 2017;39:2200-7.

12. Xin J, Liu X, Sun H, et al. A laryngoscopy-based classification system for perioperative abnormal vocal cord movement in thyroid surgery. J Int Med Res 2014;42:1029-37.

13. Yuan Q, Wu G, Hou J, et al. Correlation Between Electrophysiological Changes and Outcomes of Vocal Cord Function in 1764 Recurrent Laryngeal Nerves with Visual Integrity During Thyroidectomy. Thyroid 2020;30:739-45.

14. Youden WJ. Index for rating diagnostic tests. Cancer 1950;3:32-5.

15. Costantini O. Basic Principles of Cardiac Electrophysiology. Med Clin North Am 2019;103:767-74.

16. Del Vecchio A, Negro F, Felici F, et al. Associations between motor unit action potential parameters and surface EMG features. J Appl Physiol (1985) 2017;123:835-43.

17. Joliat GR, Guarnero V, Demartines N, et al. Recurrent laryngeal nerve injury after thyroid and parathyroid surgery: Incidence and postoperative evolution assessment. Medicine (Baltimore) 2017;96:e6674.

18. Gurrado A, Pasculli A, Pezzolla A, et al. A method to repair the recurrent laryngeal nerve during thyroidectomy. Can J Surg 2018;61:278-82.

19. Singer MC. Safety and feasibility of a novel recurrent laryngeal nerve monitoring technique. Laryngoscope 2018;128 Suppl 4:S1-S8.

20. Kandil E, Mohsin K, Murcy MA, et al. Continuous vagal monitoring value in prevention of vocal cord paralysis following thyroid surgery. Laryngoscope 2018;128:2429-32.

21. Bai B, Chen W. Protective Effects of Intraoperative
Nerve Monitoring (IONM) for Recurrent Laryngeal Nerve Injury in Thyroidectomy: Meta-analysis. Sci Rep 2018;8:7761.

22. Liu $X$, Zhang D, Zhang G, et al. Laryngeal nerve morbidity in 1.273 central node dissections for thyroid cancer. Surg Oncol 2018;27:A21-5.

23. Jansson S, Tisell LE, Hagne I, et al. Partial superior laryngeal nerve (SLN) lesions before and after thyroid surgery. World J Surg 1988;12:522-7.

24. Wang K, Cai H, Kong D, et al. The Identification, Preservation and Classification of the External Branch of the Superior Laryngeal Nerve in Thyroidectomy. World J Surg 2017;41:2521-9.

25. Wu CW, Liu X, Barczyński M, et al. Optimal stimulation during monitored thyroid surgery: EMG response characteristics in a porcine model. Laryngoscope 2017;127:998-1005.

26. Wu CW, Chai YJ, Dionigi G, et al. Recurrent laryngeal nerve safety parameters of the Harmonic Focus during thyroid surgery: Porcine model using continuous monitoring. Laryngoscope 2015;125:2838-45.

27. Estes C, Sadoughi B, Mauer E, et al. Laryngoscopic and stroboscopic signs in the diagnosis of vocal fold paresis. Laryngoscope 2017;127:2100-5.

28. Liu XL, Wu CW, Zhao YS, et al. Exclusive real-time monitoring during recurrent laryngeal nerve dissection in conventional monitored thyroidectomy. Kaohsiung J Med Sci 2016;32:135-41.

29. Liu MY, Chang CP, Hung CL, et al. Traction Injury of Recurrent Laryngeal Nerve During Thyroidectomy. World J Surg 2020;44:402-7.

30. Christakis I, Klang P, Talat N, et al. Long-term quality of voice is usually acceptable after initial hoarseness caused by a thyroidectomy or a parathyroidectomy. Gland Surg 2019;8:226-36.

(English Language Editors: A. Kassem and J. Chapnick)

Cite this article as: Zhao $\mathrm{Y}$, Zhao Z, Wang T, Zhang D, Dionigi G, Sun H. The area under the waveform of electromyography for monitoring the external branches of the superior laryngeal nerve during thyroid surgery. Gland Surg 2021;10(1):143-153. doi: 10.21037/gs-20-570 